УДК

DOI: https://doi.org/10.17308/kcmf.2019.21/2364

Поступила в редакцию 12.11.2019

Подписана в печать 15.11.2019

\title{
Влияние режимов электрохимического травления при одностадийном и двухстадийном формировании пористого кремния на степень окисления его поверхностных слоев в естественных условиях
}

по материалам XXIII Всероссийской конференции с международным участием «Рентгеновские и электронные спектры и химическая связь» (Воронеж, 1-4 октября 2019)

\section{(c2019 А. С. Леньшин ${ }^{\bowtie}$, К. А. Барков, Н. Г. Скопинцева, Б. Л. Агапов, Э. П. Домашевская \\ Воронежский государственный университет Университетская площадь, д. 1, 394018 Воронеж, Российская Федерация}

\begin{abstract}
Аннотация. В работе методами растровой электронной микроскопии и ультрамягкой рентгеновской эмиссионной спектроскопии были проведены исследования особенностей формирования многослойных структур пористого кремния и установлено влияние изменения плотности тока при электрохимическом травлении монокристаллических пластин кремния на фазовый состав поверхностных слоев сформированной пористой структуры.
\end{abstract}

Ключевые слова: кремний, пористый кремний, многослойные структуры, электрохимическое травление, рентгеновская эмиссионная спектроскопия.

\section{ВВЕДЕНИЕ}

Использование пористого кремния Por-Si в качестве чувствительного материала для различных сенсоров связано" с чрезвычайно большой площадью поверхности пористых структур по отношению к объему (до $500 \mathrm{~m}^{2} / \mathrm{g}$ ), высокой реакционной способностью его поверхности и фотолюминесцентными свойствами. На структурах пористого кремния продемонстрированы возможности создания газовых сенсоров и сенсоров влажности, действие которых основано на изменении значения тока, проходящего через структуру при адсорбции соответствующих молекул. Фотонные кристаллы в виде многослойных периодических систем на основе Por-Si также обладают перспективными оптическими свойствами, которые могут быть использованы для сенсорики химических и биологических объектов. Их оптические свойства чувствительны к небольшим изменениям показателя преломления в пористых слоях. Такие изменения в оптических свойствах были использованы для обнаружения различных типов ДНК, белков, бактерий и ферментов, органических паров. Многослой-

Леньшин Александр Сергеевич, e-mail: lenshinas@phys.vsu.ru ные пористые структуры также могут быть применены в качестве интерференционных фильтров в оптике для ИК, видимой или УФ-области спектра [1-5].

Изменением условий формирования пористого кремния можно добиться получения необходимых функциональных характеристик пористого слоя, таких как диаметр и расстояние между порами, удельная площадь поверхности, состав поверхности и др. [6-10]. В большинстве работ, посвященных многослойным структурам на основе Por-Si, ставится задача исследования функциональной характеристики (в зависимости от возможного применения) конкретной структуры, состав которой считается известным. Предполагается, что она не подвержена деградации, состав и свойства внутри каждого конкретного слоя стабильны и однородны, рост следующего слоя не влияет на строение предыдущего [11-13].

Целью данной работе являлось установление особенностей влияния изменений плотности тока при электрохимическом травлении кремния в процессе формирования многослойных систем Por-Si, на общий вид морфологии всего пористого слоя и фазовый состав на поверхности верхнего пористого слоя.

Контент доступен под лицензией Creative Commons Attribution 4.0 License.

The content is available under Creative Commons Attribution 4.0 License. 


\section{ЭКСПЕРИМЕНТАЛЬНАЯ ЧАСТЬ}

Многослойные структуры пористого кремния (multi-por-Si) формируются на поверхности подложек кристаллического кремния путем электрохимического анодирования в плавиковой кислоте HF при периодическом изменении плотности тока электрохимического травления (ЭХТ). Однослойные и двухслойные образцы пористого кремния были сформированы на пластинах монокристаллического кремния (КЭФ (100), $\rho=0.2 \mathrm{Ohm} \cdot \mathrm{cm}^{2}$ ) в растворе $\mathrm{HF}$, изопропилового спирта $\mathrm{C}_{3} \mathrm{H}_{7} \mathrm{OH}$ и перекиси водорода $\mathrm{H}_{2} \mathrm{O}_{2}$, взятых в соотношении $2: 2: 1$. При этом в процессе ЭХТ ступенчато изменялась плотность тока. Режимы травления приведены в табл. 1.

Исследования морфологических особенностей сечения образцов были проведены с помощью растрового электронного микроскопа JEOL JSM 6380 LV. Ультрамягкие рентгеновские эмиссионные $\mathrm{Si} \mathrm{L}_{2,3}$-спектры (УМРЭС) кремния образцов por-Si были получены на уникальном лабораторном рентгеновском спектрометремонохроматоре РСM-500, в высоком вакууме $1.5 \cdot 10^{-6} \mathrm{~mm} \mathrm{Hg}$ с энергетическим разрешением $\pm 0.3 \mathrm{eV}$. Данная методика чувствительна к локальной парциальной плотности электронных состояний атомов определенного сорта, благодаря чему возможна качественная оценка атомного строения пористого кремния и полуколичественная оценка относительного содержания фаз в данном материале [14]. В случае пористого кремния $S i L_{2,3}$ спектры отражают распределение $\mathrm{Si}(3 s+3 d)$ состояний с преобладанием Si 3s. Глубина анализа образцов составляла 30 и $120 \mathrm{~nm}$ при ускоряющем напряжении на аноде рентгеновской трубки, на который помещался исследуемый образец, 2 и 6 кV соответственно [15].

Экспериментальные УМРЭС Si $\mathrm{L}_{2,3}$ обрабатывались с помощью специальной компьютерной программы, которая позволяет формировать модельный спектр суммированием спектров эталонных фаз кремния с соответствующими весовыми коэффициентами, которые могут входить в состав пористого слоя, и определять фазовый состав полученных образцов por-Si путем подгонки модельного спектра к экспериментальному [14]. При моделировании $\mathrm{Si} \mathrm{L}_{2,3}$-спектров образцов por-Si использовались следующие эталонные спектры Si $\mathrm{L}_{2,3}$ : монокристаллического кремния c-Si; аморфного гидрированного кремния a-Si:H; низкокоординированного кремния $\mathrm{Si}(\mathrm{lc})$ с координационным числом $2.5-3$, который наблюдался в аморфных пленках Si [16];
Таблица 1. Режимы получения образцов пористого кремния

[Table 1. Parameters of porous silicon samples formation]

\begin{tabular}{|c|c|c|c|}
\hline No & $\begin{array}{c}\text { Etching } \\
\text { mode }\end{array}$ & $\begin{array}{c}\text { Anodizing } \\
\text { current } \\
\text { density, } \\
\mathrm{mA} / \mathrm{cm}^{2}\end{array}$ & Time, min \\
\hline 205 & $\begin{array}{c}\text { single } \\
\text { stage }\end{array}$ & 15 & 10 \\
\hline 206 & two-stage & 50 & 10 \\
\hline 207 & two-stage & $15 / 50$ & $5 / 5$ \\
\hline 208 & two-stage & $50 / 15$ & $5 / 5$ \\
\hline 209 & two-stage & $50 / 30$ & $5 / 5$ \\
\hline
\end{tabular}

субоксида кремния $\mathrm{SiO}_{1,3}$, и диоксида кремния $\mathrm{SiO}_{2}[16,17]$. Погрешность моделирования определялась как разница площадей под экспериментальным и смоделированным спектрами Si $\mathrm{L}_{2,3}$ и не превышала $10 \%$.

\section{РЕЗУЛЬТАТЫ И ИХ ОБСУЖДЕНИЕ}

На рис. 1 представлены РЭМ-изображения сколов образцов por-Si, полученных в одностадийных режимах ЭХТ с плотностями тока анодирования $15 \mathrm{~mA} / \mathrm{cm}^{2}$ (№ 205) и $50 \mathrm{~mA} / \mathrm{cm}^{2}$ (№ 206). Результаты рис. 1 показывают, что при увеличении плотности тока в указанном диапазоне происходит не только увеличение толщины пористого слоя и размера пор, но и происходит частичное растрескивание пористого слоя с нарушением его однородности. Так, толщина пористого слоя образца № 205, полученного в одностадийном режиме при плотности тока $j=15 \mathrm{~mA} / \mathrm{cm}^{2}$, составила $\sim 20 \mu \mathrm{m}$, а толщина пористого слоя для образца № 206, полученного при плотности тока $j=50 \mathrm{mAcm}^{2} \sim 30 \mu \mathrm{m}$ (рис. 1 ). С учетом времени травления $10 \mathrm{~min}$, скорость травления составила $~ 2 \mu \mathrm{m} / \mathrm{min}$ и $3 \mu \mathrm{m} / \mathrm{min}$ для образцов № 205 и № 206 соответственно и возросла в полтора раза при более чем трехкратном росте плотности тока.

На рис. 2 представлены РЭМ-изображения сколов образцов пористого кремния № 7-9, полученных в режимах двухстадийного травления. Образцы № 207 и 208 получены при плотности тока анодирования $j=15 / 50 \mathrm{~mA} / \mathrm{cm}^{2}$ и $j=50 / 15 \mathrm{~mA} / \mathrm{cm}^{2}$ соответственно, т. е. в режиме нарастания и убывания плотности тока ЭХТ, толщина пористого слоя образцов составила $\sim 25 \mu \mathrm{m}$. Толщина пористого слоя образца № 209, полученного в режиме двухстадийного травления $j=50 / 30 \mathrm{~mA} / \mathrm{cm}^{2}, \sim 30 \mu \mathrm{m}$. Анализ изображений 


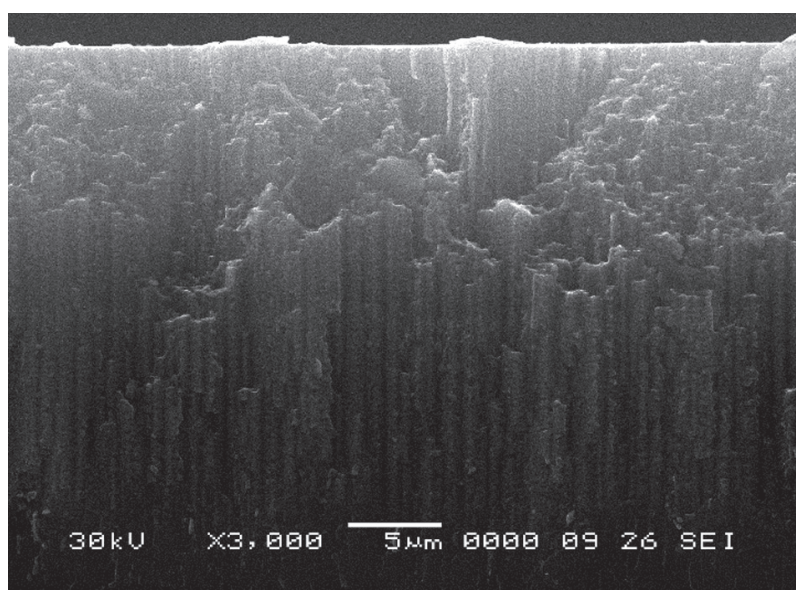

$a$

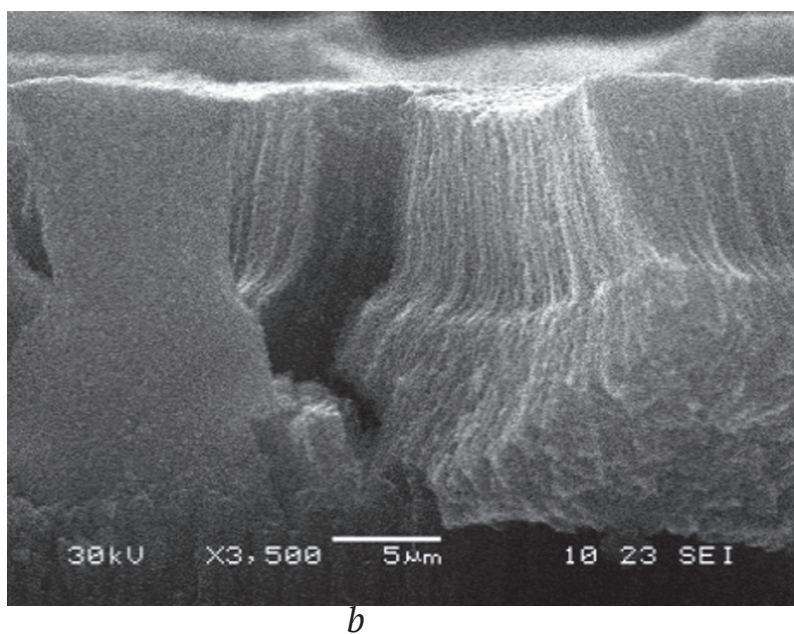

$b$

Рис. 1. РЭМ-изображения сколов образцов пористого кремния, полученных в режимах одностадийного травления: $a)$ № $\left.205 j=15 \mathrm{~mA} / \mathrm{cm}^{2} ; b\right)$ № $206 j=50 \mathrm{~mA} / \mathrm{cm}^{2}$

[Fig. 1. SEM images of cleavages of porous silicon samples obtained in single-stage etching modes: a) № $205 j=15 \mathrm{~mA} / \mathrm{cm}^{2}$; b) № $\left.206 j=50 \mathrm{~mA} / \mathrm{cm}^{2}\right]$

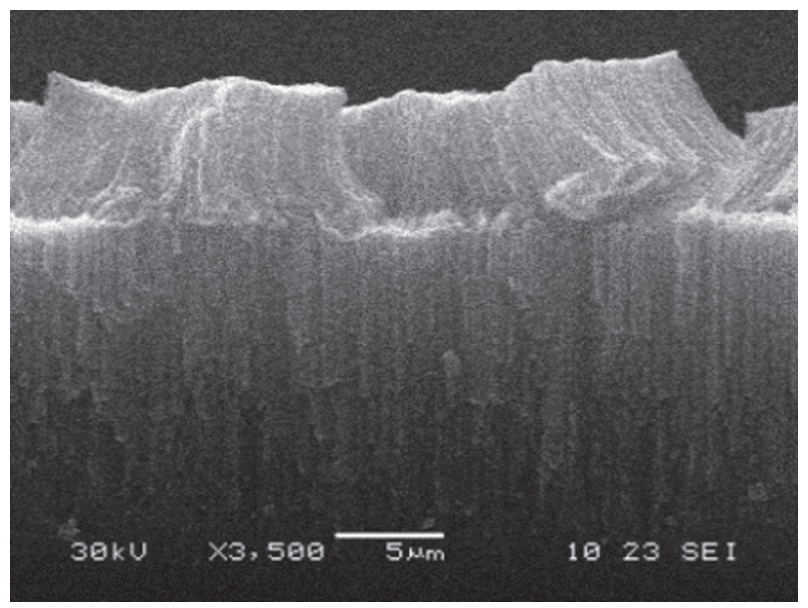

$a$

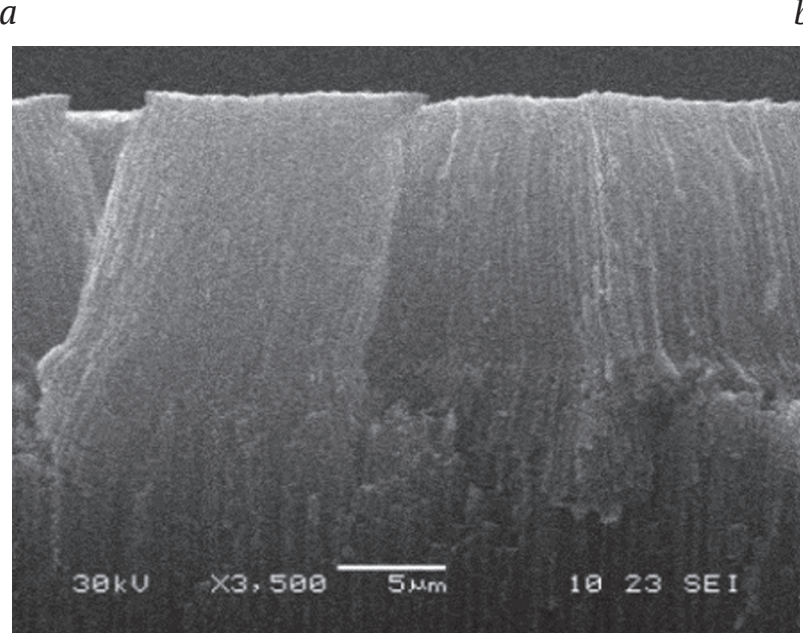

\section{$c$}

Рис. 2. РЭМ-изображения сколов образцов пористого кремния, полученных в режимах вухстадийного травления: a) № 207, $j_{a}=15 / 50 \mathrm{~mA} / \mathrm{cm}^{2} ;$ b) № $\left.208, j_{\mathrm{a}}=50 / 15 \mathrm{~mA} / \mathrm{cm}^{2} ; c\right)$ № 209, $j_{\mathrm{a}}=50 / 30 \mathrm{~mA} / \mathrm{cm}^{2}$

[Fig. 2. SEM images of cleavages of porous silicon samples obtained in two-stage etching modes:

a) № $207, j_{a}=15 / 50 \mathrm{~mA} / \mathrm{cm}^{2} ;$ b) № 208, $\left.j_{\mathrm{a}}=50 / 15 \mathrm{~mA} / \mathrm{cm}^{2} ; c\right)$ № $209, j_{\mathrm{a}}=50 / 30 \mathrm{~mA} / \mathrm{cm}^{2}$ ] 
сколов образцов позволяет сделать вывод, что глубина залегания границы между слоями структуры определяется первичным режимом ЭХТ. При этом увеличение средней плотности тока в течение всего времени ЭХТ, как и в случае одностадийного травления, приводит к формированию более развитой поверхности пористого слоя.

На рис. $3 a$ и $3 b$ приведены экспериментальные рентгеновские эмиссионные спектры $\mathrm{Si} \mathrm{L}_{2,3}$ (точки) от образцов por-Si для глубины анализа 30 и 120 nm и смоделированные спектры из эталонных фаз, полученные в результате лучшей подгонки к экспериментальным (сплошная линия). Спектры были сняты спустя 3 месяца после получения образцов. Глубина анализа методом УМРЭС, не превышающая $120 \mathrm{~nm}$, для всех образцов значительно меньше, чем толщина «верхнего» пористого слоя, поэтому, фактически, данной методикой мы исследуем изменения состава поверхности первого слоя на различных глубинах, в том числе, происходящие вследствие роста второго «нижнего» слоя, формирующегося при изменении тока ЭХТ.

Процентное соотношение фаз, полученное в результате моделирования Si L ${ }_{2,3}$ USXES-спектров всех образцов для глубин 30 и 120 nm, приведены в табл. 2 и 3. Следует отметить, что, несмотря на то, что спектры эталонных фаз [14] имеют отличительные особенности, очень часто возможно несколько вариантов разложения экспериментального спектра через сумму спектров эталонных компонент без существенного увеличения погрешности моделирования, что создает

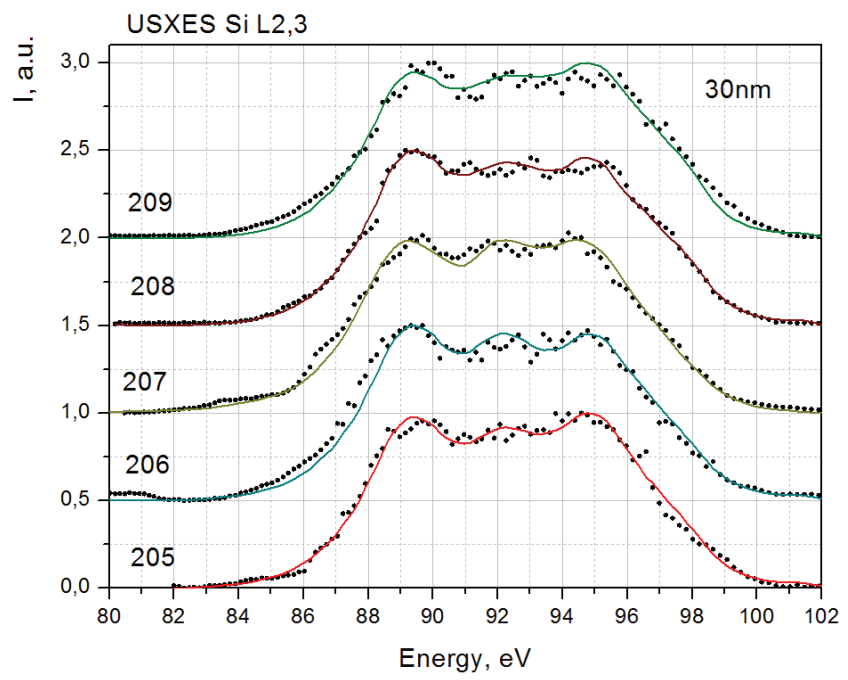

$a$ сложности при интерпретации полученных результатов. Однако во многих случаях для анализа рационально группировать результаты разложения на вклад «неокисленных» фаз, куда входят фазы аморфного, кристаллического, низкоординированного кремния и «окисленных», куда входят диоксид и субоксид кремния.

Из сопоставления данных табл. 2 и 3 видно, что для всех образцов в поверхностном слое пористого кремния (30 nm) процент содержания окисленных фаз значительно превышает количество неокисленных ( 60 к 40). Анализ фазового состава образцов на глубине порядка $120 \mathrm{~nm}$ показывает обратную ситуацию ( 40 на 60), что в целом характерно для данного материала [18], поскольку на поверхности пористого кремния взаимодействие с атмосферным кислородом происходит более активно, чем в глубине пор, и свидетельствует о том, что общие закономерности формирования пористого кремния сохраняются и в случае формирования многослойных структур.

Для анализа полученных данных были построены графические зависимости, показывающие изменения процентного соотношения окисленных и неокисленных фаз на различных глубинах анализа в зависимости от режимов получения образцов. На рис. 4 представлены графические зависимости для образцов por-Si № 206 (50 mA/cm²), № 209 (50/30 mA/cm²), № 208 $\left(50 / 15 \mathrm{~mA} / \mathrm{cm}^{2}\right)$. Значения на кривой расположены по уменьшению (рис. 4) плотности тока в процессе ЭХТ образцов.

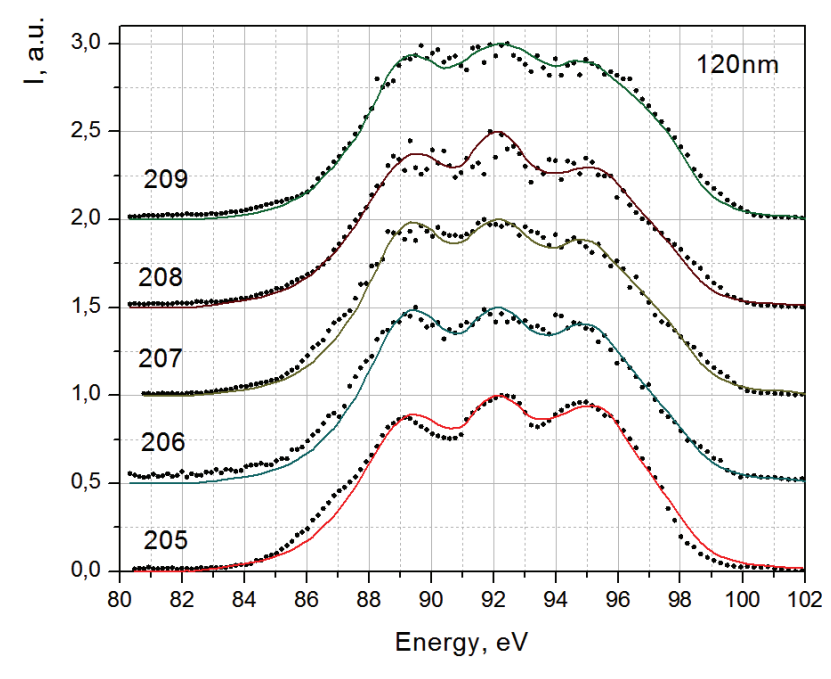

$b$

Рис. 3. USXES Si $\mathrm{L}_{2,3}$ спектры однослойных и двухслойных образцов por-Si: a) глубина анализа $30 \mathrm{~nm}$; b) $120 \mathrm{~nm}$

[Fig. 3. USXES Si L2,3 spectra of single-layer and two-layer por-Si samples:

a) depth of analysis $30 \mathrm{~nm}$; b) $120 \mathrm{~nm}$ ] 
Таблица 2. Фазовый состав образцов por-Si (глубина анализа $30 \mathrm{~nm}$ )

[Table 2. Phase composition of por-Si samples (depth of analysis $30 \mathrm{~nm}$ )]

\begin{tabular}{|c|c|c|c|c|c|c|c|c|c|}
\hline No & $\begin{array}{c}\text { Etching } \\
\text { mode, } \\
\mathrm{mA} / \mathrm{cm}^{2}\end{array}$ & $\begin{array}{c}\mathrm{a}-\mathrm{Si}, \\
\%\end{array}$ & $\begin{array}{c}\mathrm{c}-\mathrm{Si}, \\
\%\end{array}$ & $\begin{array}{c}\mathrm{a}-\mathrm{Si}(\mathrm{lc}), \\
\%\end{array}$ & $\begin{array}{c}\text { \% non- } \\
\text { oxidized } \\
\text { phases }\end{array}$ & $\begin{array}{c}\mathrm{SiO}_{1,3}, \\
\%\end{array}$ & $\begin{array}{c}\mathrm{SiO}_{2,} \\
\%\end{array}$ & $\begin{array}{c}\% \text { oxidized } \\
\text { phases }\end{array}$ & \% Err. \\
\hline 205 & 15 & 7 & 32 & - & 39 & 40 & 21 & 61 & 7 \\
\hline 206 & 50 & - & 44 & - & 44 & 42 & 14 & 56 & 9 \\
\hline 207 & $15 / 50$ & - & 45 & - & 45 & 25 & 30 & 55 & 7 \\
\hline 208 & $50 / 15$ & 9 & 25 & 8 & 42 & 58 & - & 58 & 5 \\
\hline 209 & $50 / 30$ & 15 & 14 & 14 & 43 & 41 & 16 & 57 & 9 \\
\hline
\end{tabular}

Таблица 3. Фазовый состав образцов por-Si (глубина анализа $120 \mathrm{~nm}$ )

[Table 3. Phase composition of por-Si samples (depth of analysis $120 \mathrm{~nm}$ )]

\begin{tabular}{|c|c|c|c|c|c|c|c|c|c|}
\hline No & $\begin{array}{c}\text { Etching } \\
\text { mode, } \\
\mathrm{MA} / \mathrm{cm}^{2}\end{array}$ & a-Si, \% & $\begin{array}{c}\mathrm{c}-\mathrm{Si}, \\
\%\end{array}$ & $\begin{array}{c}\mathrm{a}-\mathrm{Si}(\mathrm{lc}), \\
\%\end{array}$ & $\begin{array}{c}\text { \% non- } \\
\text { oxidized } \\
\text { phases }\end{array}$ & $\begin{array}{c}\mathrm{SiO}_{1,3}, \\
\%\end{array}$ & $\begin{array}{c}\mathrm{SiO}_{2}, \\
\%\end{array}$ & $\begin{array}{c}\% \text { oxidized } \\
\text { phases }\end{array}$ & \% Err. \\
\hline 205 & 15 & - & 49 & 8 & 57 & - & 43 & 43 & 7 \\
\hline 206 & 50 & - & 53 & - & 53 & 28 & 19 & 47 & 9 \\
\hline 207 & $15 / 50$ & - & 46 & 10 & 56 & 38 & 6 & 44 & 7 \\
\hline 208 & $50 / 15$ & 7 & 60 & - & 67 & 2 & 31 & 32 & 8 \\
\hline 209 & $50 / 30$ & 7 & 20 & 33 & 60 & 40 & - & 40 & 7 \\
\hline
\end{tabular}

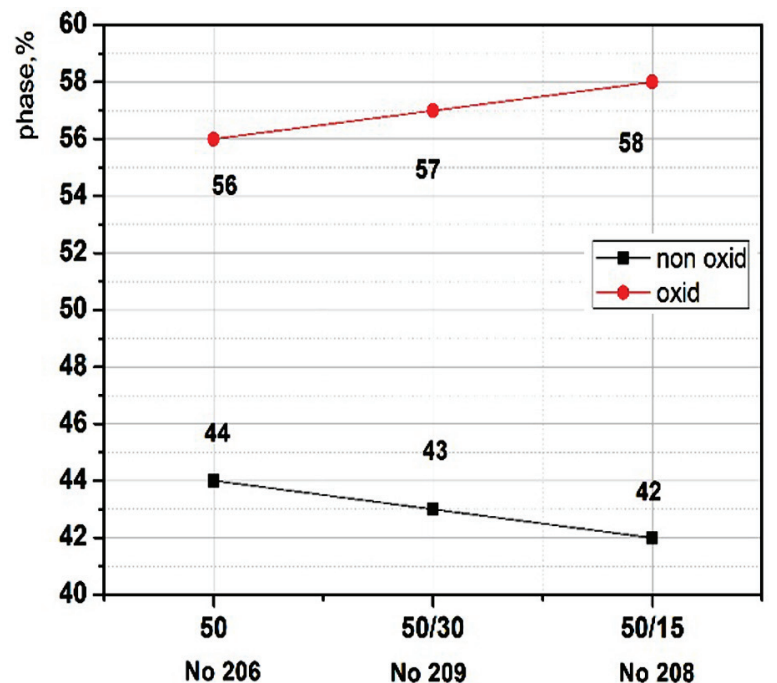

$a$

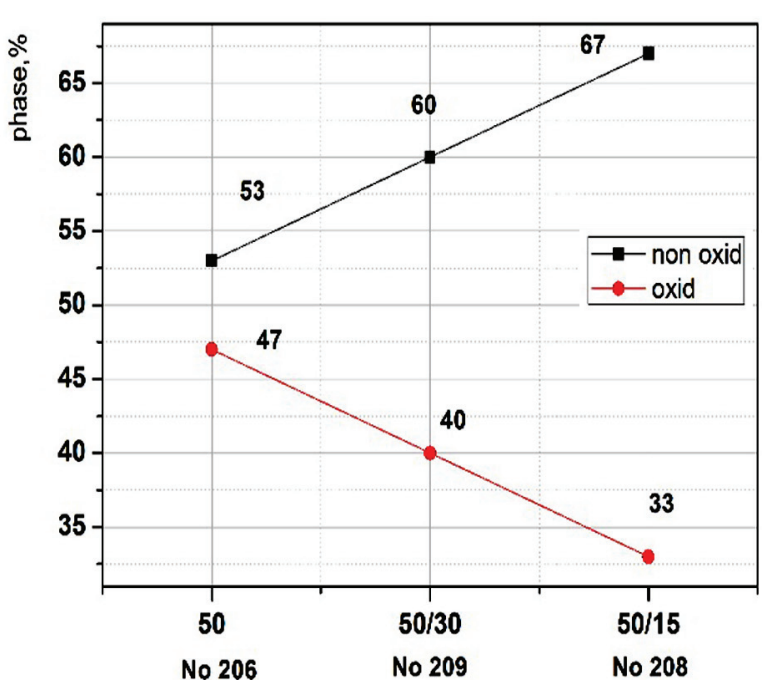

$b$

Рис. 4. Процентное соотношение окисленных и неокисленных фаз на глубине:

a) $30 \mathrm{~nm}$ и b) $120 \mathrm{~nm}$ для образцов, полученных в режимах с уменьшением тока анодирования

[Fig. 4. Percentage of oxidized and non-oxidized phases at a depth of:

a) $30 \mathrm{~nm}$ and b) $120 \mathrm{~nm}$ for samples obtained in modes with a anodization current decrease]

Из рис. $4 a$ видно, что при режимах с уменьшением плотности тока анодирования на втором этапе доля окисленных фаз на поверхности незначительно увеличивается, а доля неокисленных уменьшается. Учитывая то, что между получением образцов и их анализом методом USXES прошло около 3-х месяцев, можно предполо- жить достаточно сильное влияние естественного окисления на состав поверхностных слоев por-Si, существенно «сглаживающее» колебания состава поверхности вследствие изменения технологического режима. В то же время как в более глубоком слое (рис. $4 b$ ) доля неокисленных фаз заметно увеличивается, а доля окисленных умень- 
шается. Подобную зависимость можно объяснить тем, что в режимах с уменьшением плотности тока анодирования (для многостадийного режима) или при малой плотности тока анодирования (для одностадийного режима) диаметр пор уменьшается по глубине, что снижает доступ кислорода в более глубокие слои [19].

На рис. 5 представлены графические зависимости для образцов por-Si № $205\left(15 \mathrm{~mA} / \mathrm{cm}^{2}\right)$, № $207\left(15 / 50 \mathrm{~mA} / \mathrm{cm}^{2}\right)$, № $206\left(50 \mathrm{~mA} / \mathrm{cm}^{2}\right)$. Значения на кривой расположены по увеличению (рис. 4) плотности тока в процессе ЭХТ образцов.

При режимах формирования por-Si c увеличением плотности тока анодирования в поверхностном слое $(30 \mathrm{~nm})$ наблюдается небольшое снижение процента окисленных фаз, а в более глубоких слоях вклад оксидных фаз растет (рис. 5). При этом доля неокисленных фаз увеличивается на поверхности, но уменьшается на глубине. Данный тренд можно объяснить тем, что с увеличением плотности тока анодирования происходит формирование пор с большим диаметром, вследствие чего адсорбируемый кислород активнее проникает в более глубокие слои.

Таким образом, в работе показаны особенности формирования многослойных структур por-Si в режимах со ступенчатым изменением плотности тока ЭХТ. Показано, что морфология и фазовый состав пористого слоя как для однослойных, так и для многослойных структур отличается от поверхности к объему и зависит от

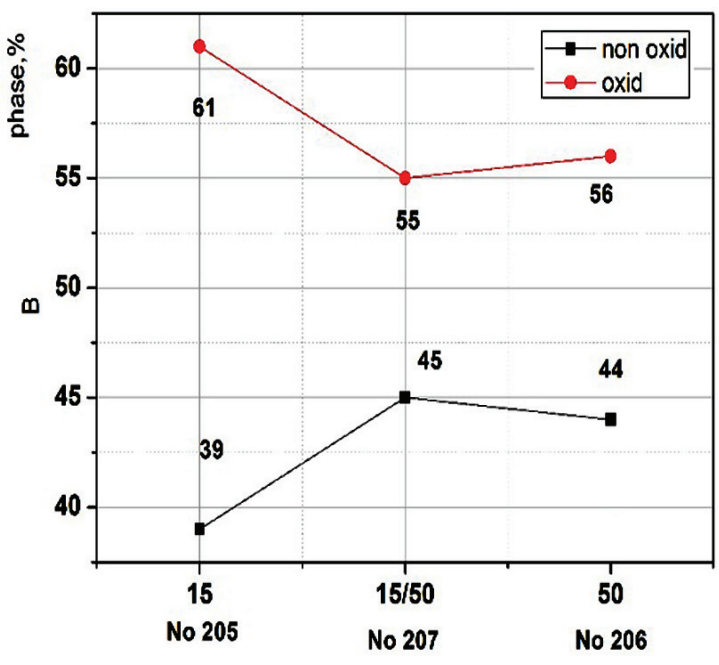

$a$ режима формирования, что необходимо учитывать при создании на основе мультислоёв por-Si устройств функциональной электроники.

\section{ИСТОЧНИК ФИНАНСИРОВАНИЯ}

Исследование выполнено при финансовой поддержке РФФИ и Правительства Воронежской области в рамках научного проекта № 1942-363004.

\section{БЛАГОДАРНОСТИ}

Результаты исследований частично получены на оборудовании Центра коллективного пользования научным оборудованием Воронежского государственного университета

\section{КОНФЛИКТ ИНТЕРЕСОВ}

Авторы декларируют отсутствие явных и потенциальных конфликтов интересов, связанных с публикацией настоящей статьи.

\section{СПИСОК ЛИТЕРАТУРЫ}

1. Moshnikov V., Gracheva I., Lenshin A., Spivak Yu. Porous silicon with embedded metal oxides for gas sensing applications // Journal of Non-Crystalline Solids, 2012 v. 358(3), pp. 590-595. DOI: https://doi. org/10.1016/j.jnoncrysol.2011.10.017

2. Pacholski C. Photonic crystal sensors based on porous silicon // Sensors, 2013, v. 13(4), pp.4694-4713. DOI: https://doi.org/10.3390/s130404694

3. Harraz F. Porous silicon chemical sensors and biosensors: A review // Sensors and Actuators B, 2014, v. 202, pp. 897-912. DOI: https://doi.org/10.1016/j. snb.2014.06.048

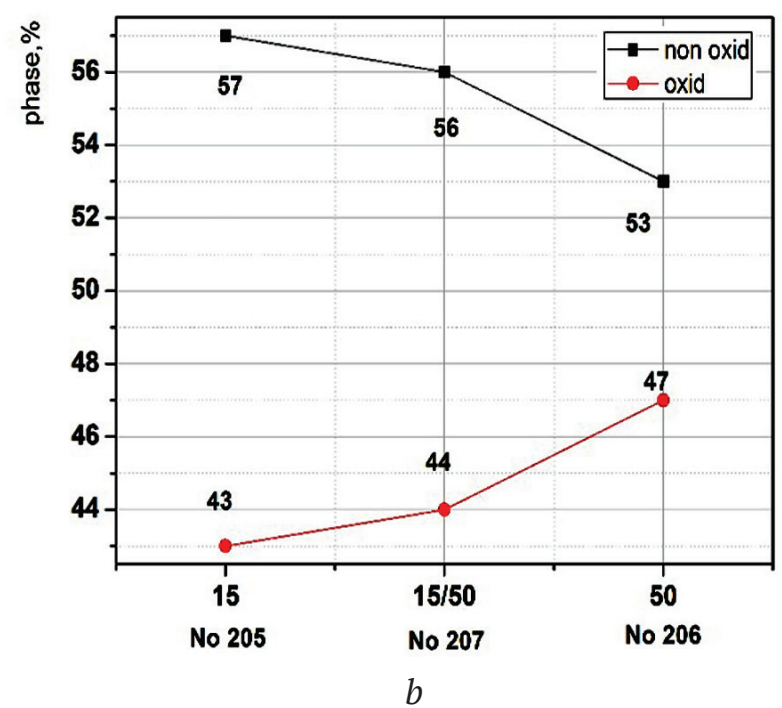

Рис. 5. Процентное соотношение окисленных и неокисленных фаз на глубине: $a) 30 \mathrm{~nm}$ и $b) 120 \mathrm{~nm}$ для образцов, полученных в режимах с увеличением тока анодирования

[Fig. 5. Percentage of oxidized and non-oxidized phases at a depth of: a) $30 \mathrm{~nm}$ and $b$ ) $120 \mathrm{~nm}$ for samples obtained in modes with a anodization current increase] 
4. Jane A., Dronov R., Hodges A., Voelcker N. Porous silicon biosensors on the Advance // Trends in Biotechnology, 2009, v. 27(4), pp. 230-239. DOI: https://doi. org/10.1016/j.tibtech.2008.12.004

5. RoyChaudhuri C. A review on porous silicon based electrochemical biosensors: beyond surface area enhancement factor // Sensors and Actuators B: Chemical, 2015, v. 10, pp. 310-323. DOI: http://dx.doi. org/10.1016/j.snb.2014.12.089

6. Canham L. Properties of porous silicon. Ed. by Canham L., Malvern: DERA, 1997, 400 p.

7. Lenshin A., Kashkarov V., Spivak Yu., Moshnikov V. Investigations of nanoreactors on the basis of p-type porous silicon: Electron structure and phase composition // Materials Chemistry and Physics, 2012, v. 135(2-3), pp. 293-297.DOI: https://doi.org/10.1016/ j.matchemphys.2012.03.095

8. Lenshin A., Kashkarov V., Turishchev S., Smirnov M., Domashevskaya E. Effect of natural aging on photoluminescence of porous silicon // Technical Physics Letters, 2011, v. 37(9), pp. 789-792. DOI: https:// doi.org/10.1134/s1063785011090124

9. Seredin P., Lenshin A., Goloshchapov D., Lukin A., Arsentyev I., Bondarev A., Tarasov I. Investigations of nanodimensional $\mathrm{Al}_{2} \mathrm{O}_{3}$ films deposited by ion-plasma sputtering onto porous silicon // Semiconductors, 2015, v. 49(7), pp. 915-920. DOI: https://doi. org/10.1134/s1063782615070210

10. Qian M., Bao X.Q., Wang L.W., Lu X., Shao J., Chen X.S. Structural tailoring of multilayer porous silicon for photonic crystal application. // Journal of Crystal Growth, 2006, v. 292(9), pp. 347-350. DOI: https://doi.org/10.1016/j.jcrysgro.2006.04.033

11. Verma D., Khan F., Singh S. Correlation between reflectivity and photoluminescent properties of porous silicon films // Solar Energy Materials \& Solar Cells, 2011, v. 95(1), pp. 30-33. DOI: https://doi. org/10.1016/j.solmat.2010.05.030

12. Theiß $\mathrm{W}$. The dielectric function of porous silicon - how to obtain it and how to use it // Thin Solid films, 1996, v. 276 (1-2), pp. 7-12. DOI: https:// doi.org/10.1016/0040-6090(95)08036-8

13. Caballero-Hernandez J., Godinho V., Lacroix B., Haro M., Jamon D., Fernandez A. Fabrication of optical multilayer devices from porous silicon coatings with closed porosity by magnetron sputtering // ACS Appl. Mater. Interfaces, 2015, v. 7(25), pp. 13889-13897. DOI: https://doi.org/10.1021/ acsami.5b02356

14. Terekhov V, Kashkarov V, Manukovskii E., Schukarev A., Domashevskaya E. Determination of the phase composition of surface layers of porous silicon by ultrasoft X-ray spectroscopy and X-ray photoelectron spectroscopy techniques // J. Electron. Spectrosc., 2001, v. 114-116, pp. 895-900. DOI: https://doi. org/10.1016/s0368-2048(00)00393-5

15. Shulakov A. X-ray emission depth-resolved spectroscopy for investigation of nanolayers. // Journal of Structural Chemistry, Supplement, 2011, v. 52(S1), pp. 1-12. DOI: https://doi.org/10.1134/s00224766 11070018

16. Mashin A., Khokhlov A., Mashin N., Domashevskaya E., Terekhov V. X-ray spectroscopic study of electronic structure of amorphous silicon and silicyne // Semiconductors, 2001, v. 35(8), pp. 956-961. DOI: https://doi.org/10.1134/1.1393035

17. Domashevskaya E., Kashkarov V., Manukovskii E., Shchukarev A., Terekhov V.XPS, USXS and PLS investigations of porous silicon// J. Electron. Spectrosc., 1998, v. 88-91, pp. 969-972. DOI: https://doi. org/10.1016/s0368-2048(97)00274-0

18. Lenshin A., Kashkarov V., Domashevskaya E., Bel'tyukov A., Gil'mutdinov F. Investigations of the composition of macro-, micro- and nanoporous silicon surface by ultrasoft X-ray spectroscopy and X-ray photoelectron spectroscopy // Applied Surface Science, 2015, 359, pp. 550-559. DOI: https://doi.org/10.1016/ j.apsusc.2015.10.140

19. Suriani Yaakob, Mohamad Abu Bakar, Jamil Ismail, Noor Hana Hanif Abu Bakar, Kamarulazizi Ibrahim. The formation and morphology of highly doped N-type porous silicon: effect of short etching time at high current density and evidence of simultaneous chemical and electrochemical dissolutions // Journal of Physical Science, 2012, v. 23(2), pp. 17-31. Available at: http://jps.usm.my/wp-content/uploads/ 2014/10/23.2.2.pdf (accessed 11.11.2019) 


\title{
Influence of Electrochemical Etching Modes under One Stage and Two Stage Formation of Porous Silicon on the Degree of Oxidation of its Surface Layer under Natural Conditions
}

based on the materials of the XXIII All-Russian Conference with International Participation "X-ray and electron spectra and chemical bond"

(Voronezh, 1-4 October 2019)

\author{
(C2019 A. S. Lenshin ${ }^{\bowtie}$, K. A. Barkov, N. G. Skopintseva, B. L. Agapov, E. P. Domashevskaya \\ Voronezh State University \\ 1, Universitetskaya pl., 394018 Voronezh, Russian Federation
}

\begin{abstract}
Purpose. In this study, some features of the formation of the multilayer structures in porous silicon were investigated by scanning electron microscopy and X-ray emission spectroscopy. Methods and tesniques. Method of electrochemical etching in hydrofluoric acid solutions was used for the synthesis of substrates. Multi-layered structures were formed by the change of the current density during electrochemical etching (ECE). The morphology of the samples was studied by scanning electron microscopy (SEM). Phase composition of the samples at the different depth of analysis was investigated by ultrasoft X-ray emission spectroscopy (USXES). Computer simulation of the obtained emission spectra was performed using the spectra of references phases.

Results. Multi-layered structures of porous silicon were obtained by the electrochemical etching technique at the step change of the current density in the ECE process. Using the SEM technique, pore sizes and the thickness of the formed porous silicon layers were determined. Analysis of the samples by USXES technique with the following computer simulation enabled an understanding of the regularities in the change of the phase composition for the samples of multi-layered por-Si structures of their formation regimes.

Conclusion. Features of formation of the multi-layered por-Si structures at the step changes of the current density in ECE process were established. The morphology and phase composition of the porous layer for both single-layer and multilayer structures differed from the surface to the volume and depended on the regime of formation. This finding must be taken into account when creating functional electronics based on por-Si multilayers.
\end{abstract}

Keywords: silicon, porous silicon, multilayer structures, electrochemical etching, X-ray emission spectroscopy.

\section{SOURCE OF FINANCING}

The study was supported by RFBR and the Government of the Voronezh Region in the framework of the scientific project no. 19-42363004 "Formation and functional characteristics of multilayer systems based on porous silicon"."

The research results were partially obtained using the equipment of the Centre for Collective Use of Scientific Equipment of Voronezh State University.

\section{CONFLICT OF INTEREST}

The authors declare the absence of obvious and potential conflicts of interest related to the publication of this article.

\footnotetext{
Alexander S. Lenshin, e-mail: lenshinas@phys.vsu.ru
}

\section{REFERENCES}

1. Moshnikov V., Gracheva I., Lenshin A., Spivak Yu. Porous silicon with embedded metal oxides for gas sensing applications. Journal of Non-Crystalline Solids, 2012 v. 358(3), pp. 590-595. DOI: https://doi. org/10.1016/j.jnoncrysol.2011.10.017

2. Pacholski C. Photonic crystal sensors based on porous silicon. Sensors, 2013, v. 13(4), pp. 4694-4713. DOI: https://doi.org/10.3390/s130404694

3. Harraz F. Porous silicon chemical sensors and biosensors: A review. Sensors and Actuators B, 2014, v. 202, pp. 897-912. DOI: https://doi.org/10.1016/j. snb.2014.06.048

4. Jane A., Dronov R., Hodges A., Voelcker N. Porous silicon biosensors on the Advance. Trends in Biotechnology, 2009, v. 27(4), pp. 230-239. DOI: https://doi. org/10.1016/j.tibtech.2008.12.004 
5. RoyChaudhuri C. A review on porous silicon based electrochemical biosensors: beyond surface area enhancement factor. Sensors and Actuators B: Chemical, 2015, v. 10, pp. 310-323. DOI: http://dx.doi. org/10.1016/j.snb.2014.12.089

6. Canham L. Properties of porous silicon. Ed. by Canham L., Malvern: DERA, 1997, 400 p.

7. Lenshin A., Kashkarov V., Spivak Yu., Moshnikov V. Investigations of nanoreactors on the basis of p-type porous silicon: Electron structure and phase composition. Materials Chemistry and Physics, 2012, v. 135(2-3), pp. 293-297. DOI: https://doi.org/10.1016/ j.matchemphys.2012.03.095

8. Lenshin A., Kashkarov V., Turishchev S., Smirnov M., Domashevskaya E. Effect of natural aging on photoluminescence of porous silicon. Technical Physics Letters, 2011, v. 37(9), pp. 789-792. DOI: https://doi. org/10.1134/s1063785011090124

9. Seredin P., Lenshin A., Goloshchapov D., Lukin A., Arsentyev I., Bondarev A., Tarasov I. Investigations of nanodimensional $\mathrm{Al}_{2} \mathrm{O}_{3}$ films deposited by ion-plasma sputtering onto porous silicon. Semiconductors, 2015, v. 49(7), pp. 915-920. DOI: https://doi. org/10.1134/s1063782615070210

10. Qian M., Bao X.Q., Wang L.W., Lu X., Shao J., Chen X.S. Structural tailoring of multilayer porous silicon for photonic crystal application. Journal of Crystal Growth, 2006, v. 292(9), pp. 347-350. DOI: https://doi.org/10.1016/j.jcrysgro.2006.04.033

11. Verma D., Khan F., Singh S. Correlation between reflectivity and photoluminescent properties of porous silicon films. Solar Energy Materials \& Solar Cells, 2011, v. 95(1), pp. 30-33. DOI: https://doi. org/10.1016/j.solmat.2010.05.030

12. Theiß W. The dielectric function of porous silicon - how to obtain it and how to use it. Thin Solid films, 1996, v. 276 (1-2), pp. 7-12. DOI: https://doi. org/10.1016/0040-6090(95)08036-8

13. Caballero-Hernandez J., Godinho V., Lacroix B., Haro M., Jamon D., Fernandez A. Fabrication of optical multilayer devices from porous silicon coatings with closed porosity by magnetron sputtering. ACS Appl. Mater. Interfaces, 2015, v. 7(25), pp. 13889-13897. DOI: https://doi.org/10.1021/acsami.5b02356

14. Terekhov V, Kashkarov V, Manukovskii E., Schukarev A., Domashevskaya E. Determination of the phase composition of surface layers of porous silicon by ultrasoft X-ray spectroscopy and X-ray photoelectron spectroscopy techniques. J. Electron. Spectrosc., 2001, v. 114-116, pp. 895-900. DOI: https://doi. org/10.1016/s0368-2048(00)00393-5

15. Shulakov A. X-ray emission depth-resolved spectroscopy for investigation of nanolayers. Journal of Structural Chemistry, Supplement, 2011, v. 52(S1), pp. 1-12. DOI: https://doi.org/10.1134/s00224766 11070018

16. Mashin A., Khokhlov A., Mashin N., Domashevskaya E., Terekhov V. X-ray spectroscopic study of electronic structure of amorphous silicon and silicyne. Semiconductors, 2001, v. 35(8), pp. 956-961. DOI: https://doi.org/10.1134/1.1393035

17. Domashevskaya E., Kashkarov V., Manukovskii E., Shchukarev A., Terekhov V.XPS, USXS and PLS investigations of porous silicon. J. Electron. Spectrosc., 1998, v. 88-91, pp. 969-972. DOI: https://doi. org/10.1016/s0368-2048(97)00274-0

18. Lenshin A., Kashkarov V., Domashevskaya E., Bel'tyukov A., Gil'mutdinov F. Investigations of the composition of macro-, micro- and nanoporous silicon surface by ultrasoft X-ray spectroscopy and X-ray photoelectron spectroscopy. Applied Surface Science, 2015, 359, pp. 550-559. DOI: https://doi.org/10.1016/ j.apsusc. 2015.10.140

19. Suriani Yaakob, Mohamad Abu Bakar, Jamil Ismail, Noor Hana Hanif Abu Bakar, Kamarulazizi Ibrahim. The formation and morphology of highly doped N-type porous silicon: effect of short etching time at high current density and evidence of simultaneous chemical and electrochemical dissolutions. Journal of Physical Science, 2012, v. 23(2), pp. 17-31. Available at: http://jps.usm.my/wp-content/uploads/ 2014/10/23.2.2.pdf (accessed 11.11.2019)
Леньшин Александр Сергеевич - к. ф.-м. н., с. н. с., кафедра физики твердого тела и наноструктур, Воронежский государственный университет, Воронеж, Российская Федерация; e-mail: lenshinas@phys.vsu.ru. ORCID iD: https://orcid. org/0000-0002-1939-253X.

Барков Константин Александрович - аспирант, кафедра физики твердого тела и наноструктур, Воронежский государственный университет, Воронеж, Российская Федерация; e-mail: barkov@phys.vsu.ru. ORCID iD: https://orcid. org/0000-0001-8290-1088.
Alexander S. Lenshin - Cand. Sci. (Phys.- Math.), Senior Researcher, Department of Solid State Physics and Nanostructures, Voronezh State University, Voronezh, Russian Federation; e-mail:lenshinas@ phys.vsu.ru. ORCID iD: https://orcid.org/00000002-1939-253X.

Konstantin A. Barkov - postgraduate student, Department of Solid State Physics and Nanostructures, Voronezh State University, Voronezh, Russian Federation; e-mail: barkov@phys.vsu.ru. ORCID iD: https://orcid.org/0000-0001-8290-1088. 
Скопинцева Наталья Геннадиевна - студент, кафедра физики твердого тела и наноструктур, Воронежский государственный университет, Boронеж, Российская Федерация; e-mail: skopintseva@phys.vsu.ru.

Агапов Борис Львович - к. т. н., Центр коллективного пользования научным оборудованием, Воронежский государственный университет, Воронеж, Российская Федерация; e-mail: b.agapov2010@yandex.ru

Домашевская Эвелина Павловна - д. ф.-м. н., профессор, заведующий кафедрой физики твердого тела и наноструктур, Воронежский государственный университет, Воронежский государственный университет, Воронеж, Российская Федерация; e-mail: ftt@phys.vsu.ru. ORCID iD: https://orcid.org/0000-0002-6354-4799.
Natalya G. Skopintseva - student, Department of Solid State Physics and Nanostructures, Voronezh State University, Voronezh, Russian Federation; email:skopintseva@phys.vsu.ru.

Boris L. Agapov - Cand. Sci. (Eng.), Centre for Collective Use of Scientific Equipment, Voronezh State University, Voronezh, Russian Federation; e-mail: b.agapov2010@yandex.ru

Evelina P. Domashevskaya - Dr. Sci. (Phys.Math.), Full Professor, Head of the Department of Solid State Physics and Nanostructures, Voronezh State University, Voronezh, Russian Federation; email: ftt@phys.vsu.ru. ORCID iD: https://orcid. org/0000-0002-6354-4799. 